\title{
Static Properties of Neutron-Rich and Proton-Rich Isotopes
}

\section{Zari Binesh*}

Department of Nuclear Physics, Faculty of Physics and Nuclear Engineering, Shahrood University of Technology, Shahrood, Iran

*Corresponding author: Zari Binesh, Department of Nuclear Physics, Faculty of Physics and Nuclear Engineering, Shahrood University of Technology, Shahrood, Iran, Tel: 09102878009;

\section{Research Article}

Volume 4 Issue 2

Received Date: September 28, 2020

Published Date: October 28, 2020

Email: binesh_z@yahoo.com

\section{Abstract}

In this paper, we have investigated some static properties of ${ }^{9} \mathrm{Be},{ }^{9} \mathrm{~B},{ }^{13} \mathrm{C},{ }^{13} \mathrm{~N},{ }^{17} \mathrm{O},{ }^{17} \mathrm{~F},{ }^{21} \mathrm{Ne}$, and ${ }^{21} \mathrm{Na}$ isotopes. The ground state and excited state energy of these isotopes have determined in the relativistic shell model and compared with experimental data. The calculated charge radius of them is in good agreement with experimental results.

Keywords: Charge radius; Energy levels

\section{Introduction}

Investigating neutron-rich or proton-rich isotopes are one of the interesting subjects in nuclear science. These isotopes have some single nucleon out of the closed core in shell structure [1]. Determining the energy levels, charge radius and other static properties of nuclei, is one of the useful components to cognition the nuclear structure [2]. Studying the nucleon-nucleon interaction is given many important properties of multi-nucleon systems [3]. The multinucleon systems can be investigated in cluster structure [1]. Recently, the cluster model is applied to studying several different systems [3]. These theories have been modeled by mathematic equations like Schrodinger equation in nonrelativistic approach and Dirac equation for spin- $1 / 2$ particle and Klein-Gordon equation for spin-1 particles in relativistic approach [4]. Many nucleon-nucleon potentials such as Woods-Saxon potential [5,6], Frost-Musulin potential [7], Pöschl-Teller potential [8] and Eckart potential [1,9] are being used by different researchers.

${ }^{9} \mathrm{Be}$ is only stable isotopes of beryllium and strongly deformed [10]. The cluster structure of ${ }^{9} B e$ is interesting for studying the dynamics of reactions with weakly bound nuclei and also nuclear astrophysics [10]. ${ }^{9} B$ has a structure similar to ${ }^{9} \mathrm{Be}$, with the odd-neutron replaced by the odd-proton [11].
There are two stable isotopes of carbon: ${ }^{12} \mathrm{C}$ and ${ }^{13} \mathrm{C} .{ }^{13} \mathrm{C}$ is used for instance in organic chemistry research, studies into molecular structures, metabolism, food labeling, air pollution and climate change. It is also used in breath tests to determine the presence of the helicobacter pylori bacteria which causes stomach ulcer. ${ }^{13} \mathrm{C}$ can also be used for the production of the radioisotope ${ }^{13} \mathrm{~N}$ which is a PET isotope $[12,13]$.

Natural nitrogen consists of two stable isotopes, ${ }^{14} \mathrm{~N}$, and ${ }^{15} \mathrm{~N}$. Fourteen radioactive isotopes have also been found so far. The ${ }^{13} \mathrm{~N}$ decays with a half-life of ten minutes to ${ }^{13} \mathrm{C}$, emitting a positron [14]. Naturally occurring oxygen is composed of three stable isotopes, ${ }^{16} \mathrm{O},{ }^{17} \mathrm{O}$, and ${ }^{18} \mathrm{O}$ with ${ }^{16} \mathrm{O}$ being the most abundant. ${ }^{17} \mathrm{O}$ is a low-abundant, natural, stable isotope of oxygen [15]. Fluorine has 18 known isotopes, with atomic masses ranging from ${ }^{14} \mathrm{~F}$ to ${ }^{31} \mathrm{~F} .{ }^{19} \mathrm{~F}$ is the only stable isotope of fluorine [16].

Sodium is the sixth most common element on Earth [17]. It is used as a heat exchanger in some nuclear reactors, and as a reagent in the chemicals industry. ${ }^{23} \mathrm{Na}$ is the only stable isotopes of sodium [18]. The Earth's crust contains 2.6\% sodium, making it the fifth most abundant metal, behind aluminum, iron, calcium, and magnesium [19]. ${ }^{23} \mathrm{Na}$ is created in the carbon-burning process in stars by fusing two carbon atoms together [20]. 
Neon is a chemical element and the second-lightest noble gas, after helium and very common element in the universe and solar system, but it is rare on earth $[17,21]$. Stable isotopes of neon are produced in stars. Neon has three stable isotopes, ${ }^{20} \mathrm{Ne},{ }^{21} \mathrm{Ne}$ and ${ }^{22} \mathrm{Ne}$ [21].
The ${ }^{9} \mathrm{Be},{ }^{9} \mathrm{~B},{ }^{13} \mathrm{C},{ }^{13} \mathrm{~N},{ }^{17} \mathrm{O},{ }^{17} \mathrm{~F},{ }^{21} \mathrm{Ne}$, and ${ }^{21} \mathrm{Na}$ isotopes can be modeled as a doubly magic $(\mathrm{N}=\mathrm{Z})$ with one additional nucleon out of core [22]. So, these isotopes can be investigated such as single particle in shell model. The experimental energy levels of these isotopes are shown in Figure 1 [23].

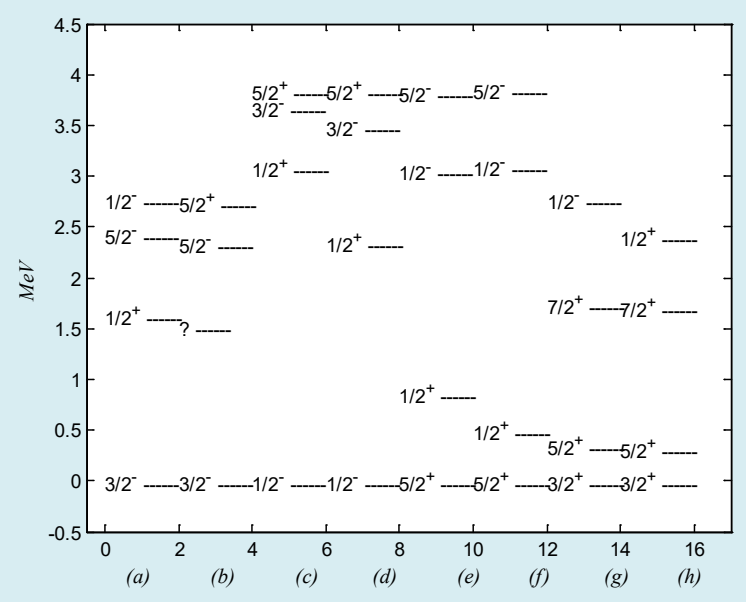

Figure 1: The experimental energy levels of a) $\left.\left.\left.\left.\left.\left.{ }^{9} \mathrm{Be}, b\right){ }^{9} \mathrm{~B}, \mathrm{c}\right){ }^{13} \mathrm{C}, \mathrm{d}\right){ }^{13} \mathrm{~N}, e\right){ }^{17} \mathrm{O}, f\right){ }^{17} \mathrm{~F}, \mathrm{~g}\right)^{21} \mathrm{Ne}, \mathrm{h}{ }^{21} \mathrm{Na}$.

In this paper, we have calculated the energy levels and charge radius of ${ }^{9} \mathrm{Be},{ }^{9} \mathrm{~B},{ }^{13} \mathrm{C},{ }^{13} \mathrm{~N},{ }^{17} \mathrm{O},{ }^{17} \mathrm{~F},{ }^{21} \mathrm{Ne}$, and ${ }^{21} \mathrm{Na}$ isotopes by solving the Dirac equation using Eckert potential plus coulomb potential for interaction between single nucleon and core cluster.

\section{The Ground State and Excited State Energy of Isotopes}

The Dirac equation is one of the most significant equations in physics [24]. There is the exact solution of this equation just only for a few simple interactions. So, the kinds of various methods have been used for the solution of this equation, exemplar, the super symmetric method [24], Nikiforov-Uvarov method $[8,25]$ and so on. By submitting suitable potential in spin symmetry Dirac equation can be written as:

$\left[\frac{d^{2}}{d r^{2}}-\frac{k(k+1)}{r^{2}}-\frac{1}{\hbar^{2} c^{2}}\left(M c^{2}+E_{n_{r}, k}\right)\left(M c^{2}-E_{n_{r}, k}+8 V_{0} \frac{e^{-2 \alpha r}}{\left(1-e^{-2 \alpha r}\right)^{2}}+\frac{2 V_{1}}{r}\right)\right] \mathrm{F}_{n_{r}, k}(r)=0$

In equation (1), $V_{0}$ and $V_{1}$ are the actual parameter describing the potential well depth and the parameter $\alpha$ representing the potential range.

By suitable approximation and $s=e^{-2 \alpha x}$ equation 1 can be written as equation 2

$$
\frac{d^{2} F}{d s^{2}}+\frac{1-s}{s(1-s)} \frac{d F}{d s}+\frac{1}{s^{2}(1-s)^{2}}\left(-\xi_{1} s^{2}+\xi_{2} s-\xi_{3}\right) F=0
$$

Where $\xi_{\mathrm{i}}$ are defined like bellow:

$$
\begin{aligned}
& \xi_{1}=-\frac{E^{2}-\mathrm{M}^{2} \mathrm{C}^{4}}{4 \alpha^{2} \hbar^{2} \mathrm{C}^{2}} \\
& \xi_{2}=-2 \frac{E^{2}-\mathrm{M}^{2} \mathrm{C}^{4}}{4 \alpha^{2} \hbar^{2} \mathrm{C}^{2}}-8 \frac{E+\mathrm{MC}^{2}}{4 \alpha^{2} \hbar^{2} \mathrm{C}^{2}} \mathrm{~V}_{0}+4 \frac{E+\mathrm{MC}^{2}}{4 \alpha^{2} \hbar^{2} \mathrm{C}^{2}} \alpha \mathrm{V}_{1} \\
& \xi_{3}=\mathrm{k}(\mathrm{k}+1)-\frac{E^{2}-\mathrm{M}^{2} \mathrm{C}^{4}}{4 \alpha^{2} \hbar^{2} \mathrm{C}^{2}}+4 \frac{E+\mathrm{MC}^{2}}{4 \alpha^{2} \hbar^{2} \mathrm{C}^{2}} \alpha \mathrm{V}_{1}
\end{aligned}
$$

(3)

By applying parametric Nikiforov-Uvarov method [22,25-28] the energy Eigen-value formula can be written as

$$
\left(n+\frac{1}{2}\right)^{2}+\frac{1}{4}+(2 n+1)\left(\sqrt{\xi_{1}-\xi_{2}+\xi_{3}+\frac{1}{4}}+\sqrt{\xi_{3}}\right)-\xi_{2}+2 \xi_{3}+2 \sqrt{\xi_{1}-\xi_{2}+\xi_{3}+\frac{1}{4}} \sqrt{\xi_{3}}=0
$$

(4)

The ground state and excited state energy of ${ }^{9} B e,{ }^{9} B$, ${ }^{13} \mathrm{C},{ }^{13} \mathrm{~N},{ }^{17} \mathrm{O},{ }^{17} \mathrm{~F},{ }^{21} \mathrm{Ne}$, and ${ }^{21} \mathrm{Na}$ isotopes are compared with experimental results in Table 1. 
Physical Science \& Biophysics Journal

\begin{tabular}{|c|c|c|c|c|c|c|}
\hline Isotopes & $\alpha(\mathbf{f m}-1)$ & $\mathrm{v}_{0}$ & $v_{1}$ & State & $\mathrm{E}_{\text {our }}(\mathrm{Mev})$ & $E_{\text {exp }}(M e v)[29]$ \\
\hline \multirow{2}{*}{ 9Be } & \multirow{2}{*}{0.0289} & \multirow{2}{*}{0.069} & \multirow{2}{*}{-410.821} & $1 \mathrm{p}_{3 / 2}$ & -58.1659 & -58.164 \\
\hline & & & & $1 f_{5 / 2}$ & -55.7804 & -55.735 \\
\hline \multirow{2}{*}{$9 \mathrm{~B}$} & \multirow{2}{*}{0.0288} & \multirow{2}{*}{0.07} & \multirow{2}{*}{-414.427} & $1 p_{3 / 2}$ & -56.3134 & -56.3136 \\
\hline & & & & $1 f_{5 / 2}$ & -53.9537 & -53.9686 \\
\hline \multirow{2}{*}{$13 \mathrm{C}$} & \multirow{2}{*}{0.0399} & \multirow{2}{*}{2.2256} & \multirow{2}{*}{-1595.6} & $1 p_{1 / 2}$ & -97.1324 & -97.1326 \\
\hline & & & & $2 p_{3 / 2}$ & -93.4231 & -93.4486 \\
\hline \multirow{2}{*}{$13 N$} & \multirow{2}{*}{0.0398} & \multirow{2}{*}{2.269} & \multirow{2}{*}{-1610.18} & $1 \mathrm{p}_{1 / 2}$ & -94.1469 & -94.1467 \\
\hline & & & & $2 p_{3 / 2}$ & -90.4895 & -90.6447 \\
\hline \multirow{2}{*}{170} & \multirow{2}{*}{0.0435} & \multirow{2}{*}{2.8859} & \multirow{2}{*}{-1679.01} & $1 d_{5 / 2}$ & -131.7623 & -131.7624 \\
\hline & & & & $2 \mathrm{p}_{1 / 2}$ & -128.6877 & -128.707 \\
\hline \multirow{2}{*}{$17 \mathrm{~F}$} & \multirow{2}{*}{0.043} & \multirow{2}{*}{2.902} & \multirow{2}{*}{-1698.53} & $1 d_{5 / 2}$ & -128.2352 & -128.2353 \\
\hline & & & & $2 p_{1 / 2}$ & -125.155 & -125.1313 \\
\hline \multirow{2}{*}{$21 \mathrm{Ne}$} & \multirow{2}{*}{0.0322} & \multirow{2}{*}{0.108} & \multirow{2}{*}{-482.271} & $1 d_{3 / 2}$ & -167.404 & -167.4047 \\
\hline & & & & $1 g_{7 / 2}$ & -165.2153 & -165.6597 \\
\hline \multirow{2}{*}{$21 \mathrm{Na}$} & \multirow{2}{*}{0.032} & \multirow{2}{*}{0.128} & \multirow{2}{*}{-525.442} & $1 d_{3 / 2}$ & -163.0454 & -163.0463 \\
\hline & & & & $1 g_{7 / 2}$ & -161.1837 & -161.3303 \\
\hline
\end{tabular}

Table 1: The ground state and excited state energy of isotopes.

In spin symmetry condition, the upper wave function is achieved in to the form

$F_{n, k}(r)=N\left(e^{-2 \alpha \mathrm{r}}\right) \sqrt{\xi_{3}}\left(1-e^{-2 \alpha \mathrm{r}}\right) \sqrt{\xi_{1}-\xi_{2}+\xi_{3}+\frac{1}{4}}+\frac{1}{2} P_{n}^{\left(2 \sqrt{\xi_{3}}, 2 \sqrt{\xi_{1}-\xi_{2}+\xi_{3}+\frac{1}{4}}\right)}\left(1-2 e^{-2 \alpha \mathrm{r}}\right)$

(5)

Where $\mathrm{N}$ is the normalization constant [30], the lower component of the Dirac spinor can be calculated by equation (6)

$$
G_{n, k}(r)=\frac{h^{2} c^{2}}{E+M c^{2}}\left(\frac{d}{d r}+\frac{k}{r}\right) F_{n, k}(r)(6)
$$

And Wave function for Dirac equation can be calculated from equation (7) as

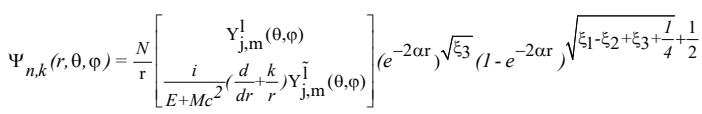

$$
\begin{aligned}
& P_{n}^{\left(2 \sqrt{\xi_{3}}, 2 \sqrt{\xi_{1}-\xi_{2}+\xi_{3}+\frac{1}{4}}\right)}\left(1-2 e^{-2 \alpha \mathrm{r}}\right)
\end{aligned}
$$

The charge radius is determined from equation (8)

$$
\left\langle r^{2}\right\rangle^{1 / 2}=\left(\frac{\int \psi_{n_{r}, k}^{*}(r) r^{2} \psi_{n_{r}, k}(r) d^{3} r}{\int \psi_{n_{r}, k}^{*}(r) \psi_{n_{r}, k}(r) d^{3} r}\right)^{1 / 2}
$$

The charge radius of isotopes is compared by experimental data in Table 2.

\begin{tabular}{|c|c|c|}
\hline Isotopes & $\left\langle r^{2}\right\rangle_{\text {our }}^{\frac{1}{2}}(\mathrm{fm})$ & $\left\langle r^{2}\right\rangle_{\text {exp }}^{\frac{1}{2}}(\mathrm{fm})$ \\
\hline${ }^{9} \mathrm{Be}$ & 2.5181 & $2.5190[30]$ \\
\hline${ }^{9} \mathrm{~B}$ & 2.5378 & $2.81[31]$ \\
\hline${ }^{13} \mathrm{C}$ & 2.4443 & $2.4614[30]$ \\
\hline${ }^{13} \mathrm{~N}$ & 2.4718 & ------ \\
\hline${ }^{17} \mathrm{O}$ & 2.6588 & $2.6932[30]$ \\
\hline${ }^{17} \mathrm{~F}$ & 2.7008 & ------ \\
\hline${ }^{21} \mathrm{Ne}$ & 2.9665 & 2.9695 \\
\hline${ }^{21} \mathrm{Na}$ & 3.0130 & $3.0136[30]$ \\
\hline
\end{tabular}

Table 2: The charge radius for ${ }^{9} \mathrm{Be},{ }^{9} \mathrm{~B},{ }^{13} \mathrm{C},{ }^{13} \mathrm{~N},{ }^{17} \mathrm{O},{ }^{17} \mathrm{~F},{ }^{21} \mathrm{Ne}$, and ${ }^{21} \mathrm{Na}$ isotopes for ground state energy.

\section{Conclusion}

In this paper, we have considered ${ }^{9} \mathrm{Be},{ }^{9} \mathrm{~B},{ }^{13} \mathrm{C},{ }^{13} \mathrm{~N},{ }^{17} \mathrm{O}$, ${ }^{17} \mathrm{~F},{ }^{21} \mathrm{Ne}$, and ${ }^{21} \mathrm{Na}$ isotopes. Since these isotopes have one additional nucleon out of core, it can be investigated as 
single particle model in relativistic shell model. Therefore, we solved the spin symmetry Dirac equation with applying PNU method. By choosing suitable potential for N-cluster interaction, the ground state and excited state energy of isotopes are obtained. These results brought in table1 and compared with experimental data.

The charge radius obtained for ground state ${ }^{9} \mathrm{Be},{ }^{9} \mathrm{~B},{ }^{13} \mathrm{C}$, ${ }^{13} \mathrm{~N},{ }^{17} \mathrm{O},{ }^{17} \mathrm{~F},{ }^{21} \mathrm{Ne}$, and ${ }^{21} \mathrm{Na}$ isotopes. As seen in table 2 our result has good agreement with experimental data.

\section{References}

1. Mousavi M, Shojaei MR (2017) Bound-state energy of double magic number plus one nucleon nuclei with relativistic mean-field approach. Pramana-J Phys 88(2): $1-5$.

2. Shojaei MR, Roshanbakht N (2016) Calculation of energy spectrum of $12 \mathrm{C}$ isotope with modified Yukawa potential by cluster models. Pramana-J Phys 87(4): 1-5.

3. Volya A, Tchuvil'sky YM (2014) Cluster properties of nuclear states in the modern shell model approach. J Phys Conf Ser 569: 1-7.

4. Mousavi M, Shojaei MR (2016) Remove Degeneracy in Relativistic Symmetries for Manning-Rosen Plus QuasiHellman Potentials by Tensor Interaction. Commun Theor Phys 66(5): 483-490.

5. Shojaei MR, Roshanbakht N (2015) Deuteron-Deuteron Cluster Model for Studying the Ground State Energy of the 4He Isotope. Chin J Phys 53(7): 1-9.

6. Urazbekov BA, Denikin AS, Sakhiev SK, Burtebaev NT (2016) Manifestation of the cluster structure of the 9Be nucleus in direct nuclear reactions. Bull Russ Acad Sci Phys 80(3): 247-251.

7. Adepoju AG, Eweh EJ (2014) Approximate and analytical bound state solutions of the Frost-Musulin potential. Can J Phys 92(1): 18-21.

8. Jia CS, Chen T, Cui LG (2009) Approximate analytical solutions of the Dirac equation with the generalized Pöschl-Teller potential including the pseudo-centrifugal term. Phys Lett A 373(18-19): 1621-1626.

9. Shojaei MR, Mousavi M (2015) Solutions of the KleinGordon equation for $l \neq 0$ with position-dependent mass for modified Eckart potential plus Hulthen potential. Int J Phys Sci 10(9): 324-328.

10. Ibraeva ET, Zhusupov MA, Dzhazairov-Kakhramanov AV, Krassovitskiy PM (2015) Inelastic $p^{9} B e$ scattering and halo-structure of excited states of ${ }^{9} \mathrm{Be}$. Nucl Phys A 933: 16-33.

11. Della Rocca V, Iachello F (2018) Cluster shell model: I. Structure of ${ }^{9} \mathrm{Be},{ }^{9} \mathrm{~B}$. Nucl Phys A 973: 1-32.

12. Staddon PhL (2004) Carbon isotopes in functional soil ecology. Trends in Ecology \& Evolution 19(3): 148-154.

13. Boutton TW (1991) Stable Carbon Isotope Ratios of Natural Materials: I. Sample Preparation and Mass Spectrometric Analysis. In: Coleman DC, Fry B (Eds.), Carbon Isotope Techniques. Academic Press, pp: 155171.

14. Cherry SR, Phelps ME (2012) Physics in Nuclear Medicine. $4^{\text {th }}$ (Edn.), Saunders, pp: 43-61.

15. Wright LE (2016) Oxygen isotopes. In: Gilbert A (Ed.), Encyclopedia of Geoarchaeology. Springer, pp: 567-574.

16. Audi G, Kondev FG, Wang M, Huang WJ, Naimi S (2017) The NUBASE2016 evaluation of nuclear properties. Chin J Phys C 41(3).

17. Emsley J (2011) Nature's Building Blocks. $2^{\text {nd }}$ (Edn.), Oxford University Press, New York, USA.

18. Audi G, Bersillon O, Blachot J Wapstra AH (2003) The Nubase evaluation of nuclear and decay properties. Nucl Phys A 729(1): 3-128.

19. Greenwood NN, Earnshaw A (1997) Chemistry of the Elements. $2^{\text {nd }}(E d n$.$) , Butterworth-Heinemann, pp: 69$.

20. Denisenkov PA, Ivanov VV (1987) Sodium synthesis in hydrogen-burning stars. Sov Astron Lett 13(3): 214.

21. Dickin AP (2005) "Neon". Radiogenic isotope geology, pp: 303.

22. Mousavi M, Shojaei MR (2017) Mirror Nuclei of 170 and $17 \mathrm{~F}$ in Relativistic and Nonrelativistic Shell Model. Adv High Energ Phys 5.

23. National Nuclear Data Center.

24. Rezvani F, Shojaei MR (2018) Relativistic effects in the study of weakly bound ${ }^{17} \mathrm{~F}$ and ${ }^{11} \mathrm{Be}$ nuclei. Pramana-J Phys 90(2): 8.

25. Rezvani F, Shojaei MR (2014) Bound states of the KleinGordon equation for spin symmetry with exponentialtype potentials by the Supersymmetry method. Journal of Theoretical Physics and Cryptography 6: 42-45.

26. Shojaei MR, Mousavi M (2016) The Effect of Tensor Interaction in Splitting the Energy Levels of Relativistic 
Physical Science \& Biophysics Journal

Systems. Adv High Energ Phys pp: 12.

27. Nikiforov AF, Uvarov VB (1988) Special Functions of Mathematical Physics. $1^{\text {st }}$ (Edn.), Birkhäuser Basel, pp: 427.

28. Ikhdair SM, Falaye BJ (2014) Bound states of spatially dependent mass Dirac equation with the Eckart potential including Coulomb tensor interaction. Eur. Phys. J. Plus 129: 1.
29. Angeli I, Marinova KP (2013) Table of experimental nuclear ground state charge radii: An update. At Data Nucl Data Tables 99(1): 69-95.

30. Mousavi M, Shojaei MR (2016) Calculation of energy and charge radius for doubly-magic nuclei of 41Ca and 41Sc with extra nucleon. Chin J Phys 54(5): 750-755.

31. Zhao Q, Ren Z, Lyu M, Horiuchi H, Funaki Y, et al. (2018) Investigation of the 9B nucleus and its cluster-nucleon correlations. Phys Rev C 97(5). 